Supporting Information

\title{
Theoretical Design of Photofunctional Molecular Aggregates for Optical Properties: An Inverse Design Approach
}

Takafumi Shiraogawa, ${ }^{*[a]}$ Masahiro Ehara*[a, b, c]

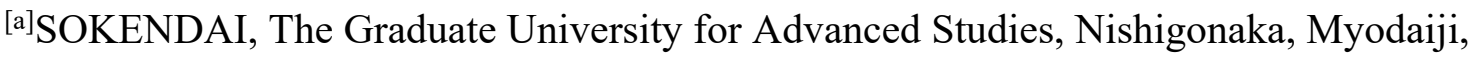
Okazaki, 444-8585, Japan.

${ }^{[b]}$ Institute for Molecular Science and Research Center for Computational Science, Nishigonaka, Myodaiji, Okazaki, 444-8585, Japan.

${ }^{[c]}$ Elements Strategy Initiative for Catalysts and Batteries (ESICB), Kyoto University, Kyoto, 615-8245, Japan. 


\section{Contents}

1. Optimization

2. Simulation of absorption and circular dichroism (CD) spectra

3. Frenkel-exciton decomposition analysis (FEDA)

4. Results

5. References 


\section{Optimization}

In the inverse design of this work, the participation coefficients must be optimized under the conditions $\sum_{A}^{N_{i}^{\mathrm{type}}} b_{i}^{(A)}=1$ and $0 \leq b_{i}^{(A)} \leq 1$. It is possible to convert the constrained optimization problem into an unconstrained one by using the following expression of the participation coefficients: ${ }^{24}$

$$
b_{i}^{(A)}=\frac{\left(t_{i}^{(A)}\right)^{2}}{\sum_{i}^{N_{i}^{\mathrm{tpe}}}\left(t_{i}^{(A)}\right)^{2}}
$$

where the constraints on $b_{i}^{(A)}$ are automatically satisfied. The arbitrary real numbers $\left\{t_{i}^{(A)}\right\}$ were optimized instead of $\left\{b_{i}^{(A)}\right\}$.

For the stop condition of the L-BFGS optimization, we employed the moderate convergence criterion of $2.20 \times 10^{-9}$ for the continuous search (see Figure 1). The quasiNewton method uses the gradient of the target property to update the molecular aggregate in the inverse design. We employed the forward finite-difference formula for the numerical differential of the target optical properties with a small number of $10^{-5}$.

The target properties of the design are the maximum positive values of the absorption and CD spectra. We initially estimated the peak with the positive maximum absorption or CD intensity in the simulated spectrum with sufficient grid points. Then, the golden section search was performed on that peak until the interval of the search becomes sufficiently small.

In the exciton model used in this work, conformational inversion of the monomer around the stacking axis does not affect the optical properties. Thus, we present the simple structures of the designed systems for simplicity. 


\section{Simulation of absorption and circular dichroism (CD) spectra}

The absorption and CD spectra can be represented as the absorption coefficient $\varepsilon$ and the molar CD $\Delta \varepsilon$, respectively, and written as follows by using overlapping Gaussian functions for each excitation:

$$
\begin{aligned}
& \varepsilon(v)=\frac{8 \pi^{3} N_{\mathrm{A}}}{3000 h c \ln 10} \frac{1}{\sqrt{\pi} \Delta v} \sum_{K}^{M} v_{0 K}\left|\mu_{0 K}\right|^{2} \exp \left\{-\left(\frac{v-v_{0 K}}{\Delta v}\right)^{2}\right\} \\
& \Delta \varepsilon(v)=\frac{32 \pi^{3} N_{A}}{3000 h c \ln 10} \frac{1}{\sqrt{\pi} \Delta v} \sum_{K}^{M} v_{0 K} R_{0 K} \exp \left\{-\left(\frac{v-v_{0 K}}{\Delta v}\right)^{2}\right\}
\end{aligned}
$$

where $h$ is the Planck's constant, $N_{A}$ is the Avogadro's number, $v$ is the photon energy, $\Delta v$ is half the bandwidth at 1/e peak height of the Gaussian, and $v_{0 K}$ is the calculated excitation energy for the transition $K \longleftarrow 0$. The ETDM and rotatory strengths are in $\mathrm{esu}^{2} \mathrm{~cm}^{2}$. 


\section{Frenkel-exciton decomposition analysis (FEDA)}

The Frenkel-exciton decomposition analysis (FEDA) method $^{49}$ enables us to qualitatively evaluate the contributions of the constitution molecules into the optical properties of the molecular aggregate. We employed this method to investigate the potential of the photofunction of the designed system. We briefly summarize the FEDA here.

The exciton electric and magnetic transition dipole moments (ETDM $\mu_{0 K}$ and MTDM $\boldsymbol{m}_{0 K}$ ) are decomposed into the individual molecular components as previously shown in equations (8) and (9), respectively:

$$
\begin{gathered}
\boldsymbol{\mu}_{0 K}=\sum_{i}^{N} \sum_{a}^{n_{i}} C_{i 0 a}^{K} \boldsymbol{\mu}_{i 0 a} \\
\boldsymbol{m}_{0 K}=\sum_{i}^{N} \sum_{a}^{n_{i}} C_{i 0 a}^{K} \boldsymbol{m}_{i 0 a} .
\end{gathered}
$$

For the absorption and emission, the exciton ETDM strength $\left|\boldsymbol{\mu}_{0 K}\right|^{2}$ can be decomposed as

$$
\left|\boldsymbol{\mu}_{0 K}\right|^{2}=\sum_{i}^{N} \sum_{a}^{n_{i}}\left(C_{i a}^{K}\right)^{2}\left|\boldsymbol{\mu}_{i 0 a}\right|^{2}+2 \sum_{i>j}^{N} \sum_{a, b}^{n_{i}, n_{j}} C_{i a}^{K} \boldsymbol{\mu}_{i 0 a} \cdot C_{j b}^{K} \boldsymbol{\mu}_{j 0 b}
$$

where the first term is the molecular intrinsic contributions from each molecule $\left(\left|\mu_{i}\right|^{2}\right)$ and the second term is the exciton ETDM interactions between the molecules $\left(\mu_{i} \mu_{j}\right)$. The FEDA allows us to analyze dividing the absorption and emission spectra of molecular aggregates into these contributions.

The rotatory strength $R_{0 K}$ shown in equation (10) represents the circular dichroism (CD) and circularly polarized luminescence (CPL):

$$
R_{0 K}=\operatorname{Im}\left(\mu_{0 K} \cdot \boldsymbol{m}_{K 0}\right) .
$$


The MTDM can be diveded into two parts in terms of the position operator of electron $\hat{r}$; these terms originate in the intrinsic and extrinsic coordinations of the constituent molecules in the reference molecular aggregate system. ${ }^{47,50}$ The magnetic dipole operator $\hat{m}$ of the MTDM (see also equation (7)) is decomposed into the corresponding terms:

$$
\begin{aligned}
\hat{m} & =\frac{i e \hbar}{2 m_{e} c} \hat{r} \times \nabla \\
& =\frac{i e \hbar}{2 m_{e} c}\left(\hat{r}^{\mathrm{int}}+\hat{r}^{\mathrm{ext}}\right) \times \nabla \\
& =\hat{m}^{\mathrm{int}}+\hat{m}^{\mathrm{ext}}
\end{aligned}
$$

where the subscriptions "int" and "ext" denote the intrinsic and extrinsic terms, respectively. The substitution of this equation into following equation (7) of the MTDM of the molecular monomer

$$
\boldsymbol{m}_{i 0 a}=\frac{i e \hbar}{2 m_{e} c}\langle i 0|\hat{m}| i a\rangle
$$

leads to

$$
\begin{aligned}
\boldsymbol{m}_{i 0 a} & =\left\langle i 0\left|\hat{m}^{\mathrm{int}}+\hat{m}^{\mathrm{ext}}\right| i a\right\rangle \\
& =\boldsymbol{m}_{i 0 a}^{\mathrm{int}}+\boldsymbol{m}_{i 0 a}^{\mathrm{ext}}
\end{aligned} .
$$

As a result, we obtain two types of MTDM of the molecular aggregate.

$$
\begin{aligned}
\boldsymbol{m}_{0 K} & =\sum_{i}^{N} \sum_{a}^{n_{i}} C_{i 0 a}^{K}\left(\boldsymbol{m}_{i 0 a}^{\mathrm{int}}+\boldsymbol{m}_{i 0 a}^{\mathrm{ext}}\right) \\
& =\boldsymbol{m}_{0 K}^{\mathrm{int}}+\boldsymbol{m}_{0 K}^{\mathrm{ext}}
\end{aligned}
$$

Then, using this equation, the rotatory strength can be written as ${ }^{50}$

$$
R_{0 K}=R_{0 K}^{\mu m}+R_{0 K}^{\mu \mu}
$$

with 


$$
\begin{aligned}
R_{0 K}^{\mu m}= & \operatorname{Im}\left(\boldsymbol{\mu}_{0 K} \cdot \boldsymbol{m}_{0 K}^{\mathrm{int}}\right) \\
= & \operatorname{Im}\left\{\sum_{i}^{N} \sum_{a}^{n_{i}}\left(C_{i a}^{K}\right)^{2} R_{i 0 a}+\sum_{i \neq j}^{N} \sum_{a, b}^{n_{i}, n_{j}} C_{i a}^{K} \boldsymbol{\mu}_{i 0 a} \cdot C_{j b}^{K} \boldsymbol{m}_{j b 0}^{\mathrm{int}}\right\} \\
R_{0 K}^{\mu \mu} & =\operatorname{Im}\left(\boldsymbol{\mu}_{0 K} \cdot \boldsymbol{m}_{0 K}^{\mathrm{ext}}\right) \\
& =-\frac{\pi\left(E_{K}-E_{0}\right)}{c h} \sum_{i>j}^{N} \sum_{a, b}^{n_{i}, n_{j}} \boldsymbol{R}_{i j} \cdot\left(C_{i a}^{K} \boldsymbol{\mu}_{i 0 a} \times C_{j b}^{K} \boldsymbol{\mu}_{j b 0}\right)
\end{aligned}
$$

where $R_{0 K}^{\mu m}$ and $R_{0 K}^{\mu \mu}$ have different physical natures which are electric-magnetic and electric-electric TDM interactions $\left(\mu m\right.$ and $\mu \mu$ ), respectively, and $\boldsymbol{R}_{i j}$ is the distance vector between the mass centers of the molecules $i$ and $j, \boldsymbol{R}_{j}-\boldsymbol{R}_{i}$. For the gauge invariance of $R_{0 K}^{\mu \mu}$, we approximated the excitation energy of the molecule by that of the molecular aggregate. The equations of $R_{0 K}^{\mu m}$ and $R_{0 K}^{\mu \mu}$ mean that the $\mu m$ and $\mu \mu$ can be decomposed into the photophysical contributions from the molecules: the rotatory strength of the molecules $\left(\mu_{i} m_{i}\right)$, electric-magnetic TDM interactions $\left(\mu_{i} m_{j}\right)$, and electricelectric TDM interactions $\left(\mu_{i} \mu_{j}\right)$ between the molecules. As a result, in the FEDA, the CD and CPL spectra are directly analyzed based on the decomposed spectra originating in these contributions of the constituent molecules. 


\section{Results}
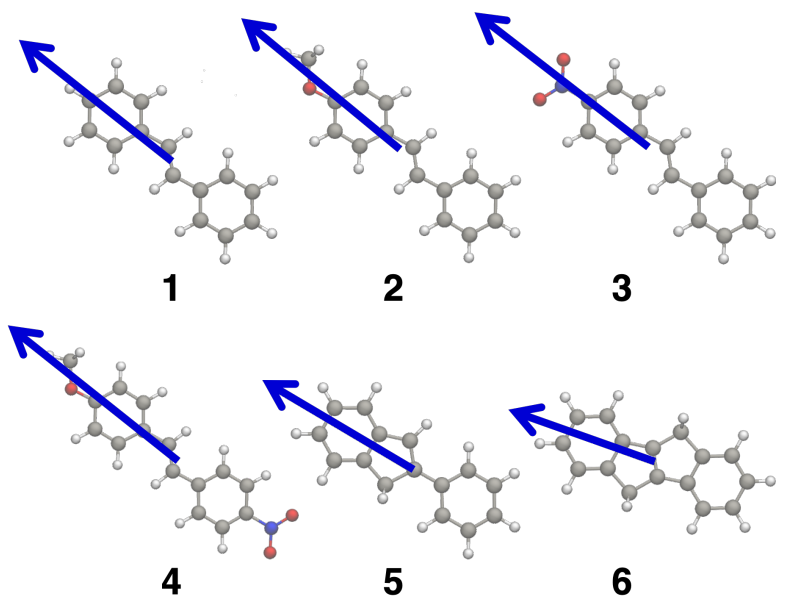

Figure S1. ETDMs of the intense electronic excitation for the first excited state of 1-6. The ETDMs are located on mass centers of the molecules.

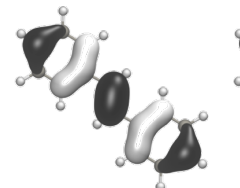

$\mathrm{H}$

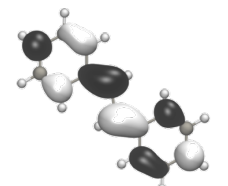

L

1

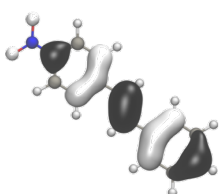

$\mathrm{H}$

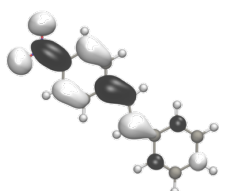

$\mathrm{L}$

3

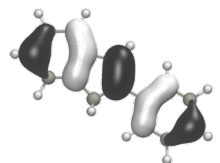

$\mathrm{H}$

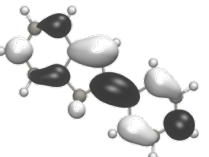

$\mathrm{L}$

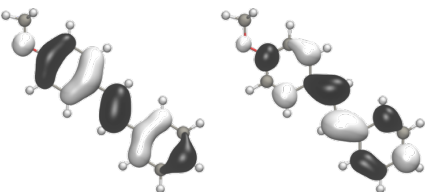

$\mathrm{H}$ 2

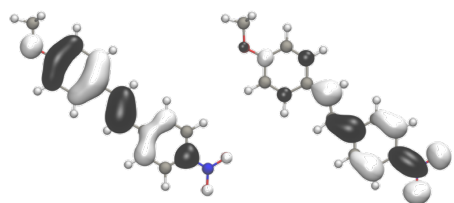

$\mathrm{H}$

4

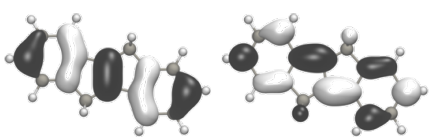

5

$\mathrm{H}$

6

Figure S2. Molecular orbitals of molecules of 1-6 (Isovalue $=|0.03|) . \mathrm{H}$ and $\mathrm{L}$ denote the highest occupied molecular orbital and the lowest unoccupied molecular orbital, respectively. 


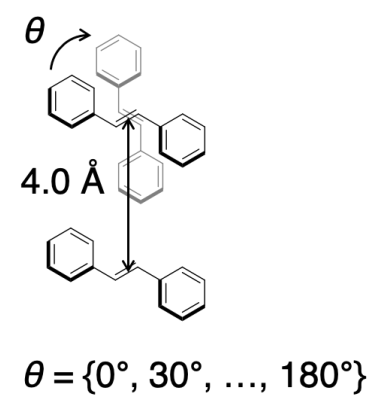

Figure S3. Simplified one-dimensional molecular aggregates composed of $\mathbf{1}$ with various conformations for examination of accuracy of the Frenkel exciton model and performance of the present inverse design approach.
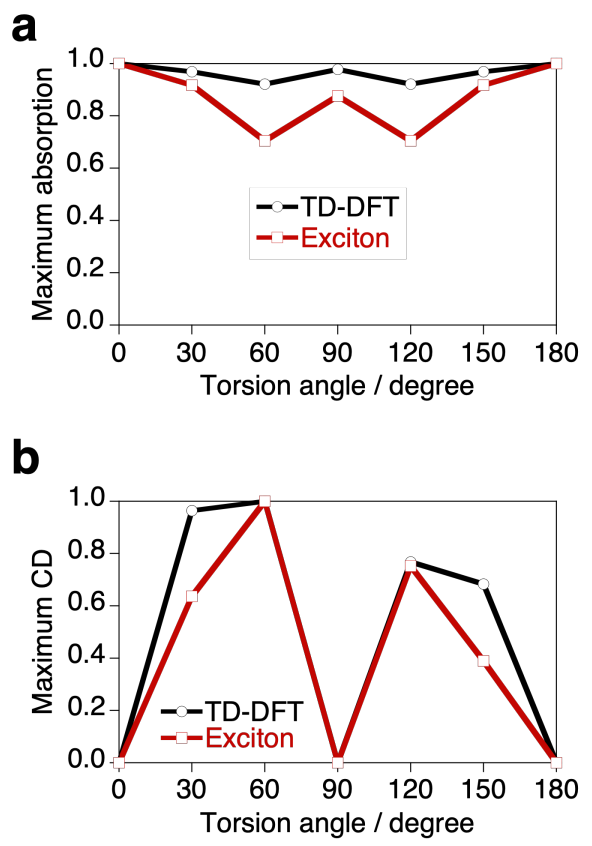

Figure S4. Torsion angle dependence of calculated maximum positive a) absorption and b) CD intensities of $\mathbf{1}$ dimer by the Frenkel exciton model and TD-DFT. The absorption and $\mathrm{CD}$ intensities are normalized based on the calculated maximum. 

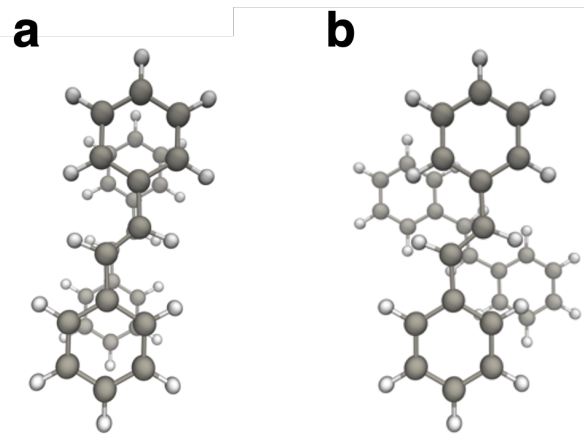

Figure S5. Structures of 1 dimers with maximum positive a) absorption and b) CD intensities.
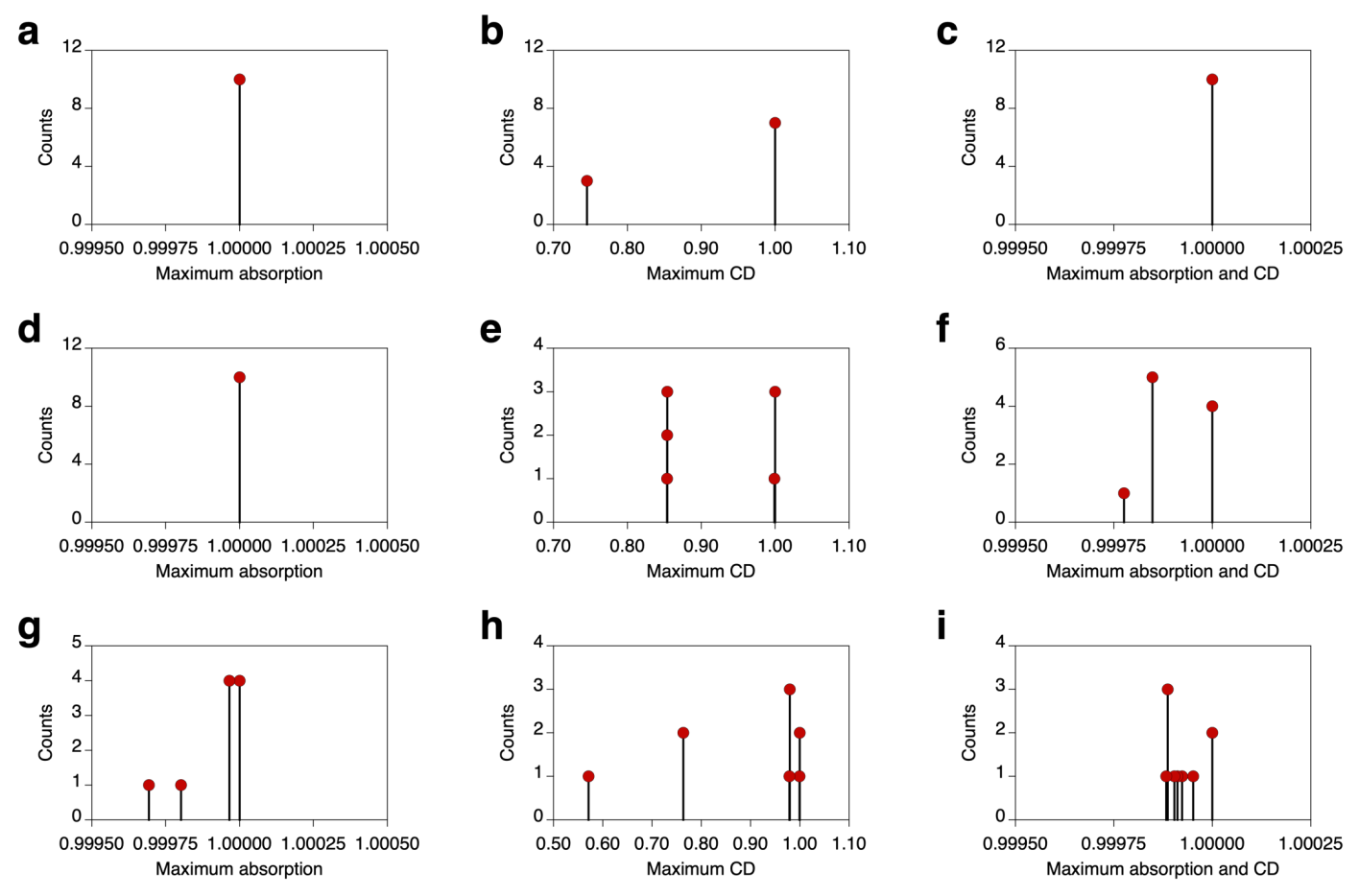

Figure S6. Results of design using ten random-initial conditions. The resulting histograms with counts of locally maximized maximum absorption and CD intensity and both of them for $(\mathrm{a}-\mathrm{c})$ dimers, $(\mathrm{d}-\mathrm{f})$ pentamers, and $(\mathrm{g}-\mathrm{i})$ decamers. The target properties are normalized based on the obtained maximum. 
2

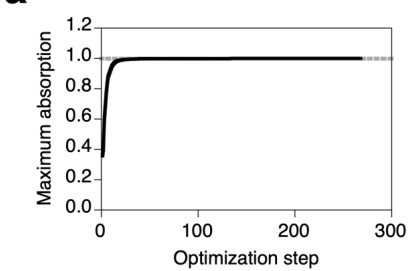

d

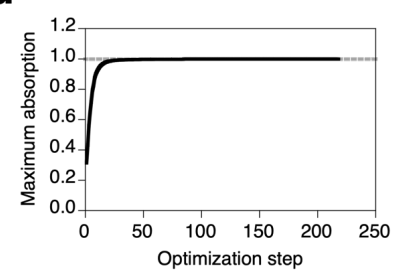

g

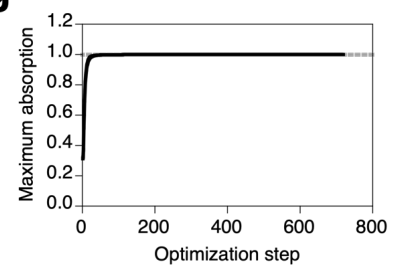

b

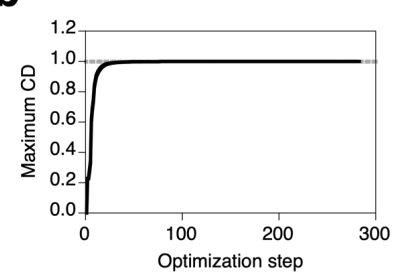

e

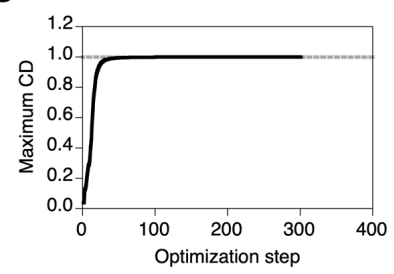

h

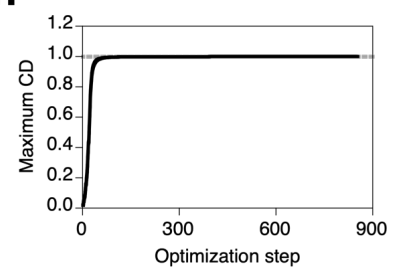

C

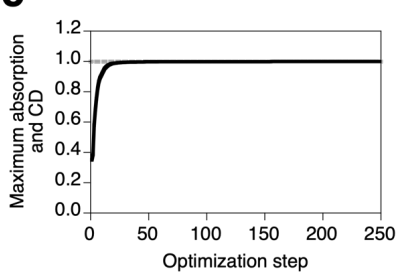

f

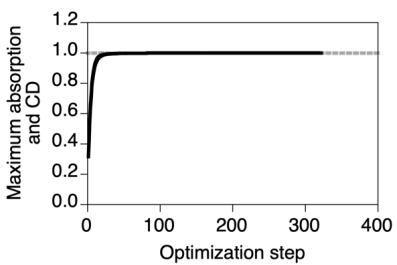

1

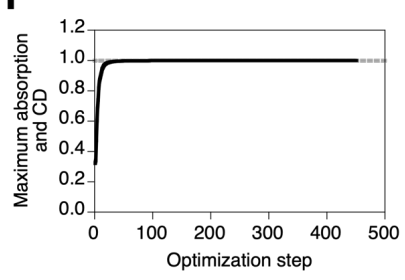

Figure S7. Optimization histories of maximum positive absorption and CD intensities and both of them for $(\mathrm{a}-\mathrm{c})$ dimers, $(\mathrm{d}-\mathrm{f})$ pentamers, and $(\mathrm{g}-\mathrm{i})$ decamers. The gray dashed lines denote the objective function after rounding off the optimized participation coefficients. The target properties are normalized based on the obtained maximum.

Table S1. Constituent molecules and structural parameters of designed dimers. The molecular site corresponds to the order of the stacking.

\begin{tabular}{ccc}
\hline $\begin{array}{c}\text { Molecular } \\
\text { site }\end{array}$ & $\begin{array}{c}\text { Molecular } \\
\text { species }\end{array}$ & $\begin{array}{c}\text { Torsion angle } \\
\left({ }^{\circ}\right)\end{array}$ \\
\hline $\begin{array}{c}\text { Absorption } \\
1\end{array}$ & 4 & 0 \\
2 & 4 & 0 \\
$\mathrm{CD}$ & & \\
1 & 4 & 0 \\
2 & 4 & 66 \\
Absorption and CD & & \\
1 & 4 & 0 \\
2 & 4 & 0 \\
\hline
\end{tabular}


Table S2. Constituent molecules and structural parameters of designed pentamers. The molecular site corresponds to the order of the stacking.

\begin{tabular}{ccc}
$\begin{array}{c}\text { Molecular } \\
\text { site }\end{array}$ & $\begin{array}{c}\text { Molecular } \\
\text { species }\end{array}$ & $\begin{array}{c}\text { Torsion angle } \\
\left({ }^{\circ}\right)\end{array}$ \\
\hline $\begin{array}{c}\text { Absorption } \\
1\end{array}$ & 4 & 0 \\
2 & 4 & 0 \\
3 & 4 & 0 \\
4 & 4 & 0 \\
5 & 4 & 0 \\
CD & & \\
1 & 4 & 0 \\
2 & 4 & 12 \\
3 & 4 & 51 \\
4 & 4 & 90 \\
5 & 4 & 102 \\
Absorption and CD & \\
1 & 4 & 0 \\
2 & 4 & 0 \\
3 & 4 & 0 \\
4 & 4 & 0 \\
5 & 4 & 0 \\
\hline
\end{tabular}


Table S3. Constituent molecules and structural parameters of designed decamers. The molecular site corresponds to the order of the stacking.

\begin{tabular}{|c|c|c|}
\hline $\begin{array}{l}\text { Molecular } \\
\text { site }\end{array}$ & $\begin{array}{c}\text { Molecular } \\
\text { species }\end{array}$ & $\begin{array}{c}\text { Torsion angle } \\
\left({ }^{\circ}\right)\end{array}$ \\
\hline \multicolumn{3}{|l|}{ Absorption } \\
\hline 1 & 2 & 0 \\
\hline 2 & 4 & 0 \\
\hline 3 & 4 & 0 \\
\hline 4 & 4 & 0 \\
\hline 5 & 4 & 0 \\
\hline 6 & 4 & 0 \\
\hline 7 & 4 & 0 \\
\hline 8 & 4 & 0 \\
\hline 9 & 4 & 0 \\
\hline 10 & 2 & 0 \\
\hline \multicolumn{3}{|l|}{$\mathrm{CD}$} \\
\hline 1 & 4 & 0 \\
\hline 2 & 4 & 9 \\
\hline 3 & 4 & 24 \\
\hline 4 & 4 & 42 \\
\hline 5 & 4 & 57 \\
\hline 6 & 4 & 72 \\
\hline 7 & 4 & 87 \\
\hline 8 & 4 & 105 \\
\hline 9 & 4 & 120 \\
\hline 10 & 4 & 129 \\
\hline \multicolumn{3}{|c|}{ Absorption and CD } \\
\hline 1 & 2 & 0 \\
\hline 2 & 4 & 0 \\
\hline 3 & 4 & 0 \\
\hline 4 & 4 & 0 \\
\hline 5 & 4 & 3 \\
\hline 6 & 4 & 354 \\
\hline 7 & 4 & 357 \\
\hline 8 & 4 & 357 \\
\hline 9 & 4 & 357 \\
\hline 10 & 2 & 357 \\
\hline
\end{tabular}


Analysis results of the absorption strength distribution of the designed systems

In this work, we restrict ourselves to the Frenkel exciton model that includes the lowest intense excitation of each molecule for simplicity. Therefore, the objective function of the design is the maximum values of the spectra in the limited excitation energy range. To examine the true maximum across the entire spectral region, we analyzed the distribution of the oscillator strength which is related to the absorption strength. Following Ref. 65, the analysis was performed based on the Thomas-Reiche-Kuhn sum rule. We employed the Frenkel exciton model for the analysis. For the dimer, we also simulated the excited states in the same excitation energy region by TD-CAM-B3LYP/6$311+\mathrm{G}(\mathrm{d})$. The TD-DFT reproduced the analysis result by the excitonic calculation. We found that all the designed systems for the strong absorption have a few percent of the total oscillator strength in the calculated energy region, which means that the remaining part lies in the higher spectral range. This result shows that the present design only targets the excited state composed by the lowest excitation of each molecule that is practically important; i.e., the global maximum peak may not have been captured in the present design. 

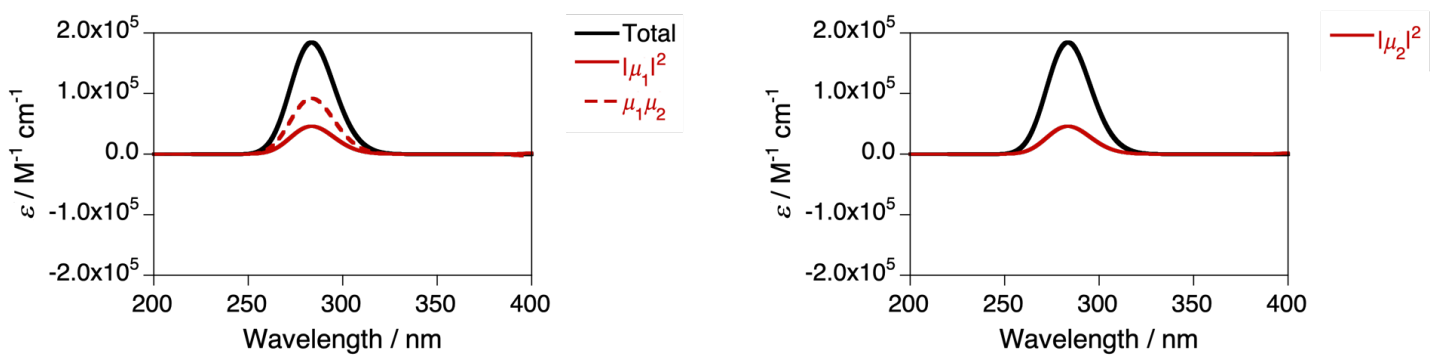

Figure S8. FEDA result of the absorption spectrum of the designed dimer for strong absorption. $\left|\mu_{i}\right|^{2}$ is the intrinsic contribution from the molecule $i . \mu_{i} \mu_{j}$ is the exciton ETDM interaction between the molecules $i$ and $j$. The indices of the molecules are in the order of the stacking.
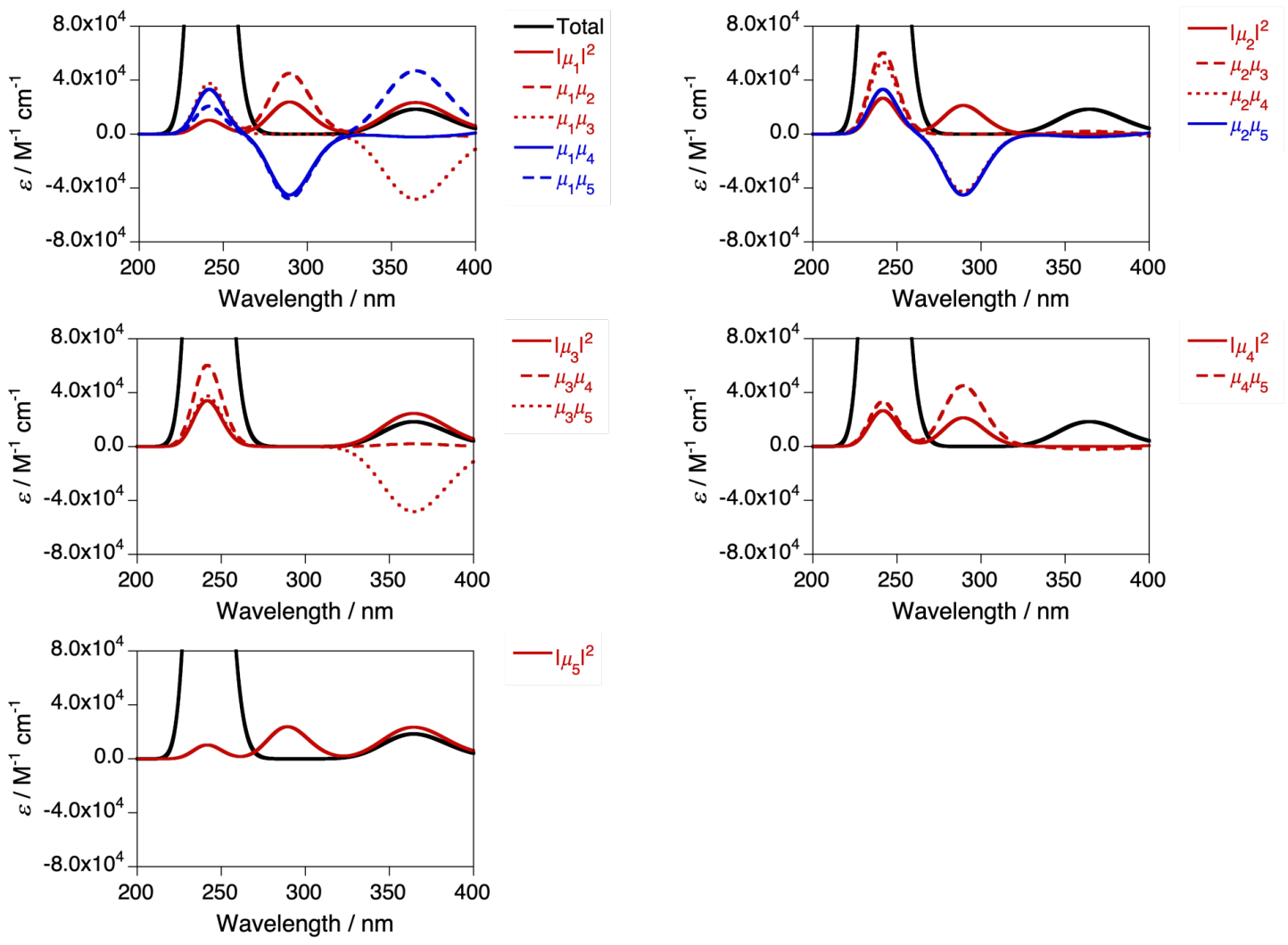

Figure S9. FEDA result of the absorption spectrum of the designed pentamer for strong absorption. $\left|\mu_{i}\right|^{2}$ is the intrinsic contribution from the molecule $i . \mu_{i} \mu_{j}$ is the exciton ETDM interaction between the molecules $i$ and $j$. The indices of the molecules are in the order of the stacking. 

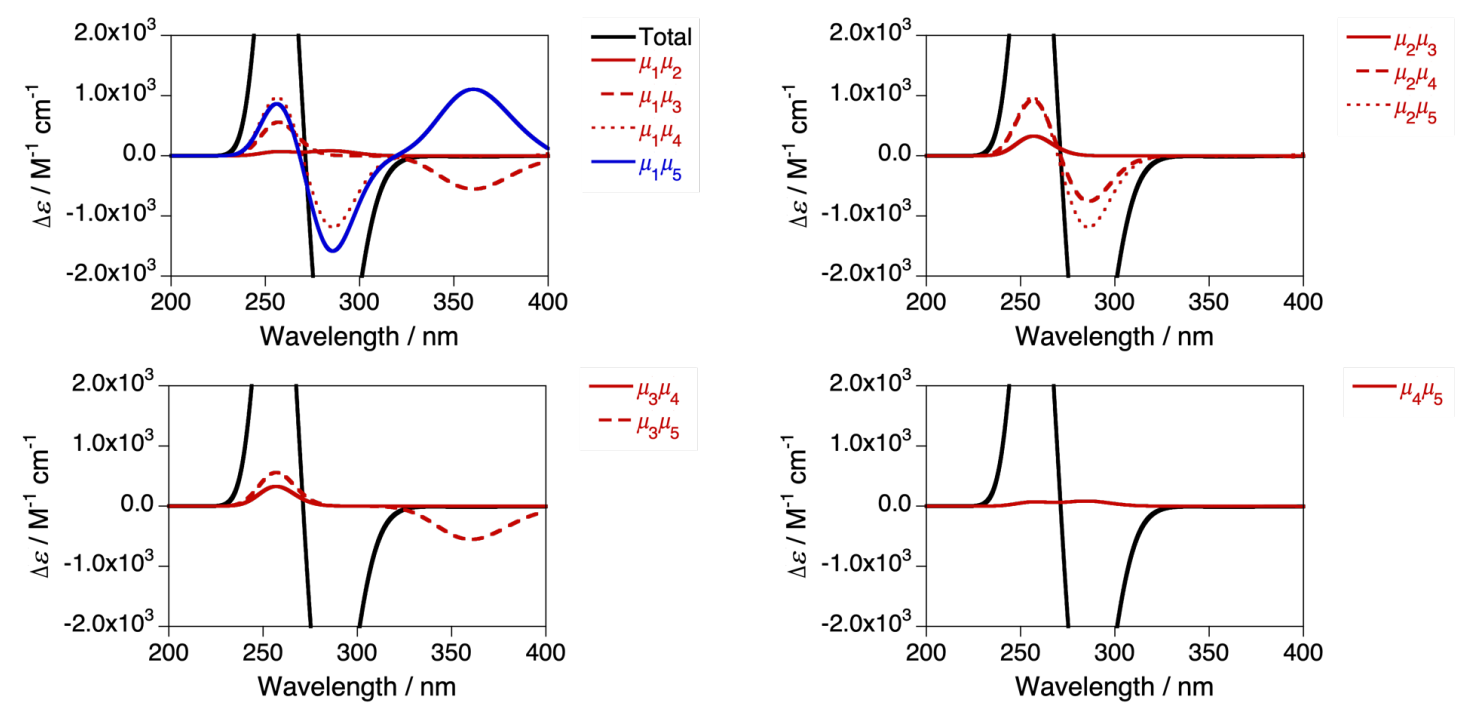

Figure S10. FEDA result of the CD spectrum of the designed pentamer for strong CD. $\mu_{i} \mu_{j}$ is the exciton ETDM interaction between the molecules $i$ and $j$. The indices of the molecules are in the order of the stacking. 

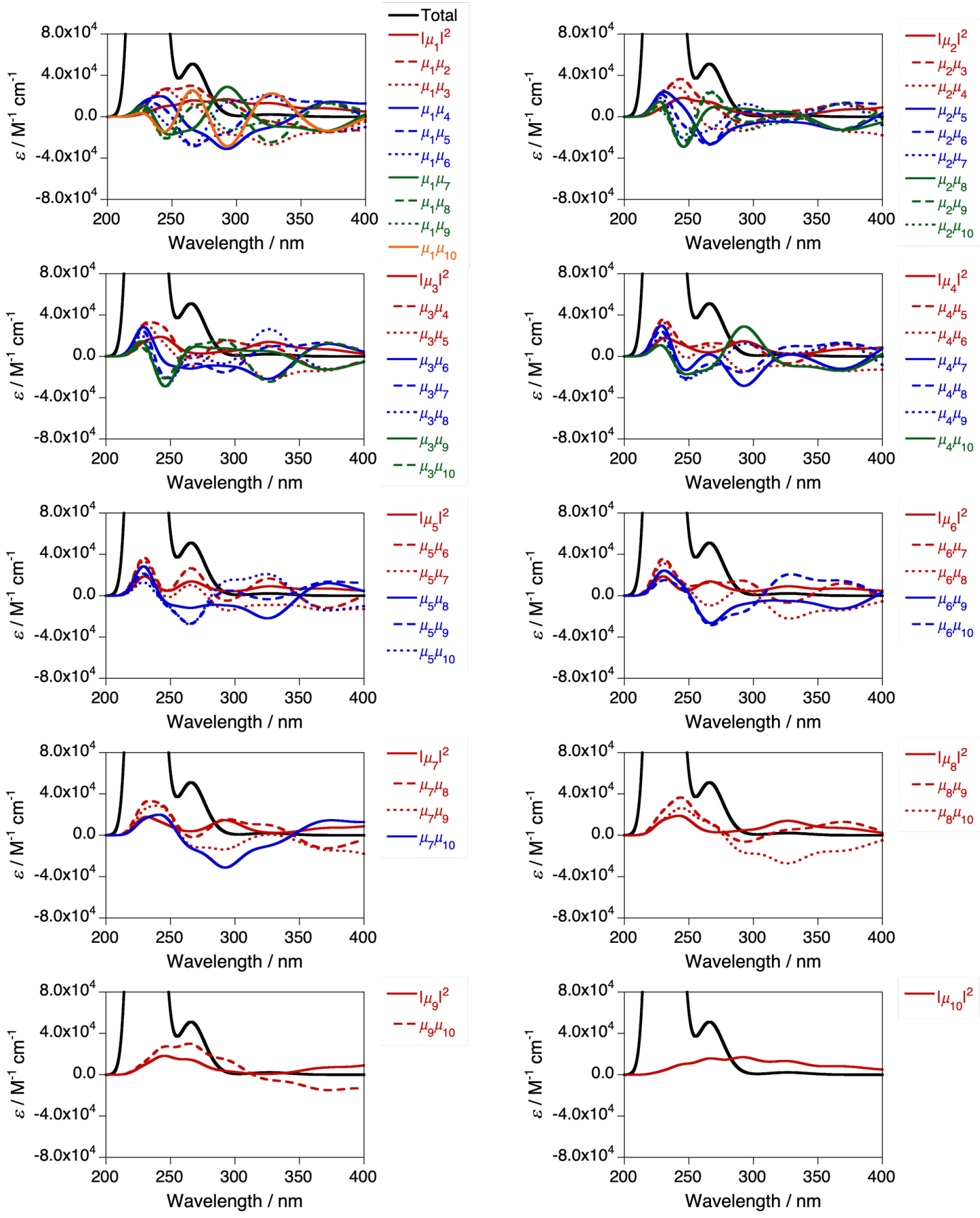

Figure S11. FEDA result of the absorption spectrum of the designed decamer for strong absorption. $\left|\mu_{i}\right|^{2}$ is the intrinsic contribution from the molecule $i . \mu_{i} \mu_{j}$ is the exciton ETDM interaction between the molecules $i$ and $j$. The indices of the molecules are in the order of the stacking. 

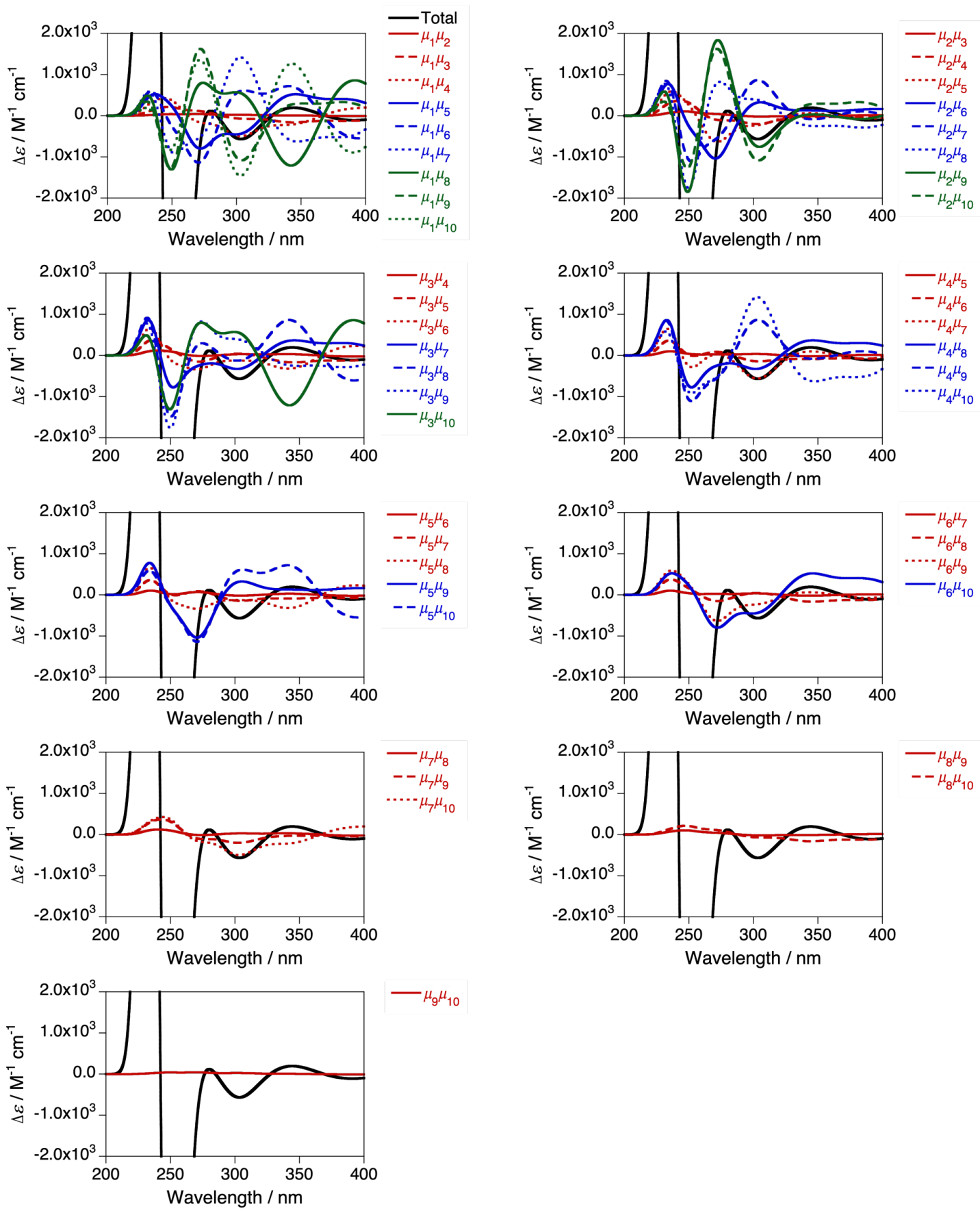

Figure S12. FEDA result of the CD spectrum of the designed decamer for strong CD. $\mu_{i} \mu_{j}$ is the exciton ETDM interaction between the molecules $i$ and $j$. The indices of the molecules are in the order of the stacking. 


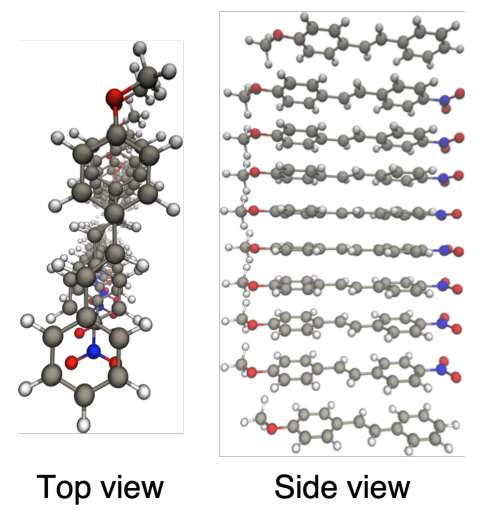

Figure S13. Designed decamer with locally maximized both of maximum positive absorption and CD intensities. 


\section{References}

(24) Wang, M.; Hu, X.; Beratan, D. N.; Yang, W., Designing Molecules by Optimizing Potentials. J. Am. Chem. Soc. 2006, 128, 3228-3232.

(47) Harada, N.; Nakanishi, K., Circular Dichroic Spectroscopy-Exciton Coupling in Organic Stereochemistry; University Science Books: Mill Valley, CA, 1983.

(49) Shiraogawa, T.; Ehara, M.; Jurinovich, S.; Cupellini, L.; Mennucci, B., FrenkelExciton Decomposition Analysis of Circular Dichroism and Circularly Polarized Luminescence for Multichromophoric Systems. J. Comput. Chem. 2018, 39, 931-935.

(50) Jurinovich, S.; Pescitelli, G.; Di Bari, L.; Mennucci, B., A TDDFT/MMPol/PCM Model for the Simulation of Exciton-Coupled Circular Dichroism Spectra. Phys. Chem. Chem. Phys. 2014, 16, 16407-16418.

(58) Frisch, M. J.; Trucks, G. W.; Schlegel, H. B.; Scuseria, G. E.; Robb, M. A.; Cheeseman, J. R.; Scalmani, G.; Barone, V.; Petersson, G. A.; Nakatsuji, H.; Li, X.; Caricato, M.; Marenich, A. V.; Bloino, J.; Janesko, B. G.; Gomperts, R.; Mennucci, B.; Hratchian, H. P.; Ortiz, J. V.; Izmaylov, A. F.; Sonnenberg, J. L.; Williams; Ding, F.; Lipparini, F.; Egidi, F.; Goings, J.; Peng, B.; Petrone, A.; Henderson, T.; Ranasinghe, D.; Zakrzewski, V. G.; Gao, J.; Rega, N.; Zheng, G.; Liang, W.; Hada, M.; Ehara, M.; Toyota, K.; Fukuda, R.; Hasegawa, J.; Ishida, M.; Nakajima, T.; Honda, Y.; Kitao, O.; Nakai, H.; Vreven, T.; Throssell, K.; Montgomery, Jr., J. A.; Peralta, J. E.; Ogliaro, F.; Bearpark, M. J.; Heyd, J. J.; Brothers, E. N.; Kudin, K. N.; Staroverov, V. N.; Keith, T. A.; Kobayashi, R.; Normand, J.; Raghavachari, K.; Rendell, A. P.; Burant, J. C.; Iyengar, S. S.; Tomasi, J.; Cossi, M.; Millam, J. M.; Klene, M.; Adamo, C.; Cammi, R.; Ochterski, J. W.; Martin, R. L.; Morokuma, K.; Farkas, O.; Foresman, J. B.; Fox, D. J. Gaussian 16, Revision B.01; Gaussian, Inc., Wallingford, CT, 2016.

(65) Zheng, L.; Polizzi, N. F.; Dave, A. R.; Migliore, A.; Beratan, D. N., Where Is the Electronic Oscillator Strength? Mapping Oscillator Strength across Molecular Absorption Spectra. J. Phys. Chem. A 2016, 120, 1933-1943. 